INPLASY

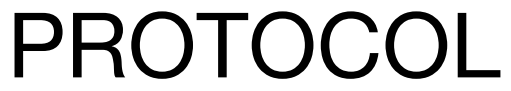

To cite: Zhang et al. Effect of Continuous Positive Airway Pressure On IL-6 Levels In Obstructive Sleep Apnea: A Meta-analysis. Inplasy protocol 2021110008. doi:

10.37766/inplasy2021.11.0008

Received: 03 November 2021

Published: 03 November 2021

Corresponding author:

Zhang Meng

zhangmeng20210706@163.com

Author Affiliation:

Dalian Medical University.

Support: National Natural

Funds.

Review Stage at time of this submission: Data extraction.

Conflicts of interest:

None declared.

\section{Effect of Continuous Positive Airway Pressure On IL-6 Levels In Obstructive Sleep Apnea: A Meta-analysis}

Zhang, M1; Wu, SQ2; Min, LF³.

Review question / Objective: Is CPAP effective on IL-6 levels in OSA patients? Systematic review and meta-analysis of randomized controlled trials.

Condition being studied: Obstructive sleep apnea hypopnea syndrome (OSAHS) is a sleep disorder that affects multiple systems of the whole body and causes multiple organ damage, especially in cardiovascular diseases, and is an independent risk factor for hypertension, coronary heart disease and respiratory failure.CPAP therapy, as the most widely recognized and effective treatment for OSA, keeps the respiratory airway open and stable through positive pressure ventilation, and can effectively reduce cardiovascular and cerebrovascular risks after long-term treatment. To better evaluate its efficacy, this meta-analysis was conducted to evaluate the effect of continuous positive airway pressure (CPAP) on the level of vasculitis biomarker IL-6 and to establish predictors of CPAP response, providing a reliable protocol for the diagnosis and evaluation of OSA treatment.

INPLASY registration number: This protocol was registered with the International Platform of Registered Systematic Review and Meta-Analysis Protocols (INPLASY) on 03 November 2021 and was last updated on 03 November 2021 (registration number INPLASY2021110008).

\section{INTRODUCTION}

Review question / Objective: Is CPAP effective on IL-6 levels in OSA patients? Systematic review and meta-analysis of randomized controlled trials.
Rationale: Evaluate Effect of Continuous Positive Airway Pressure On IL-6 Levels In Obstructive Sleep Apnea.

Condition being studied: Obstructive sleep apnea hypopnea syndrome (OSAHS) is a sleep disorder that affects multiple systems of the whole body and causes multiple 
organ damage, especially in cardiovascular diseases, and is an independent risk factor for hypertension, coronary heart disease and respiratory failure.CPAP therapy, as the most widely recognized and effective treatment for OSA, keeps the respiratory airway open and stable through positive pressure ventilation, and can effectively reduce cardiovascular and cerebrovascular risks after long-term treatment. To better evaluate its efficacy, this meta-analysis was conducted to evaluate the effect of continuous positive airway pressure (CPAP) on the level of vasculitis biomarker IL-6 and to establish predictors of CPAP response, providing a reliable protocol for the diagnosis and evaluation of OSA treatment.

\section{METHODS}

Search strategy: From inception to November 1, 2021, we will search PubMed, Embase, and the Cochrane Library. The efficacy of CPAP on interleukin-6 (IL-6) in OSA patients was evaluated by a consensus selected randomized controlled trial (RCT).

Participant or population: Get diagnosed Obstructive Sleep Apnea Hypopnea syndrome.

\section{Intervention: Receive CPAP therapy.}

Comparator: IL-6 Levels Before CPAP therapy and After CPAP therapy.

Study designs to be included: Randomized Controlled Trials or Cohort studies.

Eligibility criteria: Only trials thatObstructive Sleep Apnea Hypopnea syndrome and CPAP therapy will be included.

Information sources: PubMed, Embase, and the Cochrane Library.

Main outcome(s): IL-6 level reduce.

Data management: Use endnote.

Quality assessment / Risk of bias analysis:

$Q$ test was used to evaluate the heterogeneity of RCTs studies included in meta-analysis, and 12 statistics were used for quantitative analysis of heterogeneity.Among them, $Q$ test level indicated that there was no statistical heterogeneity when P>0.1.Otherwise, there is statistical heterogeneity. The 12 statistics can be divided into three categories: low heterogeneity $(0 \%-40 \%)$, moderate heterogeneity $(30 \%-60 \%)$, and high heterogeneity $(50 \%-90 \%)$. $12>75 \%$ was considered significant statistical heterogeneity, and the random effect model was used; otherwise, the fixed effect model was used.

Strategy of data synthesis: Use Cochrane system software Revman 5.3 versions statistical analysis.

Subgroup analysis: Significant clinical heterogeneity was treated by subgroup analysis or sensitivity analysis, or descriptive analysis only.

Sensitivity analysis: None

Language: No restriction.

Country(ies) involved: China.

Other relevant information: None.

Keywords: Continuous Positive Airway Pressure; IL-6; Obstructive Sleep Apnea; Meta-analysis.

Dissemination plans: None.

Contributions of each author:

Author 1 - ZHANG MENG - The author drafted the manuscript.

Author 2 - Wu Shuqing - The author provided statistical expertise.

Author 3 - Min LF - The author contributed to the development of the selection criteria, and the risk of bias assessment strategy. 